\section{ACCIONES EN EL FOMENTO DE LA INVESTIGACIÓN MÉDICA EN EL PERÚ}

\section{[ACTIONS TO PROMOTE MEDICAL RESEARCH IN PERU]}

\author{
Juan Arturo Rodríguez ${ }^{1, b}$, Edén Galán-Rodas²,a, \\ Manuel Núñez $z^{3, a}$, Ciro Maguiña ${ }^{4, c}$
}

Señor Editor. La temática del último número de la Revista Peruana de Medicina Experimental y Salud Pública, está dirigida a la importancia de la investigación en salud, así como a las iniciativas y reformas implementadas en los últimos años, en particular con relación al rol directriz del Instituto Nacional de Salud (INS) en la construcción y fortalecimiento del Sistema Nacional de Investigación en Salud (SNIS) ${ }^{(1,2)}$. Es en este contexto, que queremos acotar algunas acciones y estrategias que han emprendido el Colegio Médico del Perú (CMP) y el Comité Nacional de Residentado Médico (CONAREME) con el fin de contribuir y fomentar la investigación médica en el país.

En la actual gestión del CMP 2010 - 2011, se han asumido como prioridades la educación médica continua y la investigación científica. Por ello, hemos mejorado, renovado y potenciado el sistema informático, reactivado el Comité Nacional de Investigación Científica y creado el Premio Nacional a la Investigación Científica, cuya convocatoria se realizará próximamente. Se ha realizado la suscripción al Programa de acceso a la investigación en salud (HINARI) de la Organización Mundial de la Salud, y en alianza con la Organización Panamericana de la Salud, se ha implementado un programa de capacitación en búsqueda de evidencia científica en Internet dirigido en este primer año a once regiones del interior del país (Ayacucho, Madre de Dios, Piura, Tumbes, Puno, Ica, Huánuco, Cajamarca, Arequipa, San Martín y Amazonas). En esta misma línea de trabajo, se ha programado la realización de cursos talleres en redacción científica y publicación para médicos jóvenes, así como el firme compromiso de apoyo en jornadas científicas estudiantiles y congreso científico nacional de estudiantes de medicina, para el año 2011. Asimismo, se viene incorporando en la página web institucional importantes libros, manuales y guías de práctica clínica, y con el objetivo de mejorar la difusión de estos documentos se ha creado el Fondo Editorial del CMP.

El Comité Nacional de Residentado Médico (CONARE$\mathrm{ME})$, en cumplimiento de su función conductora de la

\footnotetext{
Asociación Nacional de Médicos Residentes del Perú, Lima, Perú.

Comité Médico Joven, Colegio Médico del Perú, Lima, Perú

Comité Nacional de Residentado Médico. Lima, Perú

Decanato, Colegio Médico del Perú. Lima, Perú.

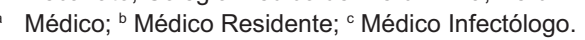

Recibido: 24-11-10 Aprobado: 09-12-10 formación de médicos especialistas ${ }^{(4)}$, ha aprobado recientemente la creación de un Premio Nacional a la Investigación realizada por médicos residentes, así como un fondo concursable destinado a la investigación sobre la formación en el residentado médico, concordantes con el establecimiento de que la investigación en recursos humanos en salud forma parte de las prioridades nacionales de investigación en salud ${ }^{(3)}$. Para facilitar el acceso a información científica relevante y actualizada, también se ha aprobado la suscripción a bases de datos de evidencia científica, las cuales estarán a disposición de universidades, hospitales y principalmente de los médicos residentes. En enero de 2011, convocaremos a todos los involucrados en el Sistema Nacional de Residentado Médico para evaluar los procesos de admisión de los últimos cinco años y determinar los próximos pasos a seguir rumbo al proceso único de admisión ${ }^{(5)}$.

Finalmente, estamos convencidos de la necesidad primordial de generar nuevos conocimientos a través de la investigación científica, proceso necesario para conocer la problemática de salud del país, y así generar soluciones y mejoras al sistema de salud, contribuyendo en última instancia en la mejor calidad de vida de la población ${ }^{(6)}$.

\section{Conflictos de Interés}

JAR, EGR y CM han tomado las decisiones o ejecutan las acciones que expresan en esta carta en relación con el CMP, JAR y MN lo han hecho en el CONAREME.

\section{REFERENCIAS BIBLIOGRÁFICAS}

1. Cabezas C. Necesidad de la investigación en salud para contribuir a la equidad, la salud y el desarrollo. Rev Perú Med Exp Salud Publica. 2010;27(3):310-11.

2. Yagui M, Espinoza M, Caballero P, Castilla T, Garro G, Yamaguchi $\mathbf{P}$, et al. Avances y retos en la construcción del sistema nacional de investigación en salud en el Perú. Rev Peru Med Exp Salud Publica. 2010;27(3):387-97.

3. Caballero P, Yagui M, Espinoza M, Castilla T, Granados A, Velásquez A, et al. Prioridades regionales y nacionales de investigación en salud, Perú 2010-2014: un proceso con enfoque participativo y descentralista. Rev Peru Med Exp Salud Publica. 2010;27(3):98-411.

4. Perú, Ministerio de Salud. Decreto Supremo № 008-88-SA. Normas básicas del sistema nacional de residentado médico. Lima: MINSA; 1988.

5. Comité Nacional de Residentado Médico. Acta de la Sesión Ordinaria de CONAREME del 22 de octubre 2010. Plan Operativo del Comité Nacional de Residentado Médico. Lima: CONAREME; 2010.

6. Villaverde $\mathbf{H}$, Seas $\mathbf{C}$. La investigación en la calidad de la formación médica: obligación o no? En: Cuaderno de debate en salud: calidad de la educación en la formación médica. Lima: Fondo Editorial del Colegio Médico del Perú; 2010. p. 32-34.

Correspondencia: Juan Arturo Rodríguez García

Dirección: Av. Malecón Armendáriz 791, Miraflores, Perú.

Correo electrónico: juanarturo502@hotmail.com 